\title{
Impact of layer defects in ferroelectric thin films
}

\author{
J. M. Wesselinowa \\ University of Sofia, Department of Physics \\ Blvd. J. Bouchier 5, 1164 Sofia, Bulgarid* \\ S.Trimper, and K. Zabrocki \\ Fachbereich Physik, Martin-Luther-Universität, D-06099 Halle Germany
}

(Dated: November 21, 2018)

\begin{abstract}
Based on a modified Ising model in a transverse field we demonstrate that defect layers in ferroelectric thin films, such as layers with impurities, vacancies or dislocations, are able to induce a strong increase or decrease of the polarization depending on the variation of the exchange interaction within the defect layers. A Green's function technique enables us to calculate the polarization, the excitation energy and the critical temperature of the material with structural defects. Numerically we find the polarization as function of temperature, film thickness and the interaction strengths between the layers. The theoretical results are in reasonable accordance to experimental datas of different ferroelectric thin films.

PACS numbers: 77.80.-e, 77.55.+f, 68.60.-p, 64.60.Cn
\end{abstract}

*Electronic address: julia@phys.uni-sofia.bg

${ }^{\dagger}$ Electronic address: trimper@physik.uni-halle.de 


\section{INTRODUCTION}

Defects in crystals influence significantly the physical properties of almost all materials. In the last decades defect engineering has been developed as a part of modern semiconductor technology. There is also an increasing interest in studying defects and their related strain fields in ferroelectrics (FE), in particular in low dimensional systems [1]. A broad variety of defects have been implemented and analyzed mainly in $\mathrm{ABO}_{3}$-type $\mathrm{FE}$, such as $\mathrm{BaTiO}_{3}$ and $\mathrm{Pb}(\mathrm{Zr}, \mathrm{Ti}) \mathrm{O}_{3}(\mathrm{PZT})$ 1, 2, 3, 4], bismuth layer-structured FE, such as $\mathrm{Bi}_{4} \mathrm{Ti}_{3} \mathrm{O}_{12}$ (BTO) [5, 6, 7, 8] as well as $\mathrm{SrBi}_{2} \mathrm{Ta}_{2} \mathrm{O}_{9}(\mathrm{SBT})$ [9, 10, 11]. To control the polarization and piezoelectric properties, PZT is often modified by various elements of lower valency ( $\mathrm{K}, \mathrm{Mn}, \mathrm{Fe})$ and higher talent (La, Nb, Ta) cations. The modified PZT are generally classified into the two categories named as "hard" and "soft" ones [12]. The substitution of lower-valent cations, compared to the constituent ions, leads to oxide vacancies which induces a soft FE behavior, whereas higher-valent substitution gives rise to cation vacancies offering a hard FE behavior. Contrary to hard FE, the soft FE are characterized by a lower coercitive field $E_{c}$, lower remanent polarization $\sigma_{r}$ and higher hysteresis losses. The different FE properties are generally originated by the occurrence of defects. In materials with confined dimensions, the influence of defects can be even more pronounced due to the enhanced relative volume of the "defective" regions [4].

FE thin films have been extensively studied due to their ability for applications in nonvolatile FE random access memories (FeRAMs) [13]. For this application the thin film ferroelectrics should reveal large remanent polarizations, low coercitive fields, and fatiguefree properties. PZT and SBT have been widely investigated as appropriate material in Fe-RAMs. Otherwise the poor fatigue characteristic and the small remanent polarization, are viewed as the major barrier for their applications.

BTO is a promising alternative to PZT, because of its large spontaneous polarization along the $a$-axis. Recently, it was reported that the substitution of small amounts of impurities, such as $\mathrm{Sm}$ and $\mathrm{Nd}$ for $\mathrm{Bi}$ and $\mathrm{V}, \mathrm{W}$ and $\mathrm{Nb}$ for $\mathrm{Ti}$ in the pseudoperowskite $\left[\left(\mathrm{Bi}_{2} \mathrm{Ti}_{3} \mathrm{O}_{10}\right)^{2-}\right]$ layers of BTO, is effective for lowering the leakage current and enhancing

the remanent polarization [6, 7, 8]. Noguchi et al. [9] reported the control of FE properties in Sr-site-modified SBT bulk ceramics, in which the Sr-site was partially substituted by La atoms, showed a lower coercitive field as the original material; but, the remanent 
polarization was also decreased in this material. On the other hand, several reports have revealed that the $\sigma_{r}$ value is enhanced in Sr-deficient and Bi-excess SBT films because of the substitution of excess $\mathrm{Bi}$ atoms in the $\mathrm{Sr}$ site [14, 15]. However, $E_{c}$ also increases in these films. Since the ionic radius of the La ion is almost the same as that of the Bi ion, the electronegativity is also important for controlling the FE properties in the site-engineering technique. In fact, the electronegativity of a $\mathrm{Bi}$ ion is much higher than that of a La ion. A similar effect was obtained in Sr-deficient and Pr-substituted SBT films [10].

PZT films are considered to most promising candidates for applications in microelectromechanical systems (MEMS) since they have large piezoelectric coefficients and electromechanical coupling coefficients. As known from bulk ceramics, the piezoelectric and dielectric properties vary substantially with the composition ( $\mathrm{Zr}$ to Ti ratio). While the bulk PZT ceramics have been well investigated, there is still a lack of understanding the piezoelectric and dielectric properties of PZT thin films, particularly as a function of composition. With increasing Ti-content, larger remanent polarization and higher coercive voltage are observed [1]. Impact of misfit dislocations on the polarization instability of epitaxial nanostructured FE perovskites (PZT nanoislands) is investigated by Chu et al. [4]. Their results suggest that misfit engineering is indispensable for obtaining nanostructured FE's with stable polarization. The properties of $\mathrm{BaTiO}_{3}$ are modified by substituting of tetravalent ions such as $\mathrm{Zr}^{4+}$ for $\mathrm{Ti}$ sites. This increases the dielectric constant and broadens the sharp temperature dependence of the dielectric constant around the Curie temperature. With the addition of strontium to $\mathrm{PbTiO}_{3}$, the phase-transition temperature decreases with the increasing Sr concentration.

Influence of defects on FE phase transitions is one of key-like topics of the modern studies of FE. Constant attention to this topic is connected with its role in the real FE problem solution from the point of views of the fundamental science as well as of the above mentioned applications. It is well known that localized spin excitations can arise in FE and magnetic systems with broken spatial translational symmetry, i.e. due to the presence of boundary surfaces, interfaces, impurities and other defects in the crystal. These localized modes appearing in the above mentioned structures are experimentally observed using the neutron and Raman scattering [16, 17], or, more recently, far infrared absorption [18]. The Green's function theories have been extensively employed to describe these modes in semi-infinite transverse Ising models [19] and FE thin films [20]. In addition to bulk spin waves there 
may be localized modes associated with the impurity layer [19] and with the surface [20]. A transfer matrix formalism is applied to study a semi-infinite Heisenberg ferromagnetic superlattice with surface cell and impurity cell at arbitrary distance from the surface [21]. The occurrence of localized modes associated with the impurity cell is demonstrated sufficiently. In the dielectric continuum approximation, Zhang et al. 222] investigated the effect of different kinds of coupling to the localized interface optical-phonon modes in two coupled semi-infinite $N$-constituent superlattices with a structural defect layer. The influence of molecular impurity ions on FE phase transitions is studied by Vikhin and Maksimova using the phenomenological Landau theory [23]. It is shown that molecular impurity ions with charge transfer-local vibration degrees of freedom which bi-linearly interact with FE order parameter can induce rather strong increasing of the critical temperature of FE phase transition at reasonable concentrations of molecular impurities. It could be topical, for instance, for the $\mathrm{KDP}: \mathrm{MnO}_{4}$ case. Using a combination of first-principles and effective-Hamiltonian approaches, Dieguez et al. 24] obtained a phase diagram in temperature and misfit strain in epitaxial $\mathrm{BaTiO}_{3}$ that is qualitatively different from that reported by Pertsev et al. [25]. In particular, in 24] is found a region of " $r$ phase" at low temperature where Pertsev et al. have reported an "ac phase".

The most of the published papers consider the defects influence on the spin excitations. A first attempt to find out the influence of defects on the polarization of $\mathrm{FE}$ thin films are elucidated by Alpay et al. [26, 27]. A thermodynamic analysis has been carried out to investigate the role of dislocations in FE materials. Due to the coupling of the stress field of the dislocation and the polarization, there is expected a drastic variation of the polarization near to the dislocation. The compressive regions enhance the polarization and increase Curie temperature whereas tensile stresses decrease polarization with a commensurate drop in the Curie temperature [26, 27]. But actually the dependence of polarization and Curie temperature on defects in FE thin films is not so intensively studied theoretically. Therefore, it is the aim of the present paper to investigate the polarization of FE thin films with defect layers in more detail. Based on the Ising model in a transverse field and using the Green's function theory we calculate the spin-wave energies, the polarization and the phase transition temperature for a FE thin film with different structural defect layers. The numerical results are compared with those of bulk materials and with thin films without defect layers. 


\section{THE MODEL AND THE MATRIX GREEN'S FUNCTION}

Let us consider a three dimensional ferroelectric system on a simple cubic lattice composed of $N$ layers in z-direction. The layers are numbered by $n=1, \ldots, N$, where the layers $n=1$ and $n=N$ represent the two surfaces of the system. The bulk is established by the remaining $(N-2)$ layers. The specific surface effects are included by additional coupling parameters between bulk and surface layers. In particular, we start with the Hamiltonian of the Ising model in a transverse field which includes both, bulk and surface properties.

$$
H=-\frac{1}{2} \sum_{i j} J_{i j} S_{i}^{z} S_{j}^{z}-\Omega_{b} \sum_{i \in b} S_{i}^{x}-\Omega_{s} \sum_{i \epsilon s} S_{i}^{x},
$$

where $S^{x}$ and $S^{z}$ are components of spin- $\frac{1}{2}$ operators, and $\Omega_{b}$ and $\Omega_{s}$ represent transverse fields in the bulk and surface layers, and the sums are over the internal and surface lattice points, respectively. $J_{i j}$ is an exchange interaction between spins at nearest-neighbor sites $i$ and $j$, and $J_{i j}=J_{s}$ between spins on the surface layer, otherwise it is $J_{b}$. We assume that one or more of the layers can be defect, since $J_{d}$ and $\Omega_{d}$ denote the exchange interaction and the transverse field of the defect layer. In the ordered phase we have the mean values $<S^{x}>\neq 0$ and $<S^{z}>\neq 0$, and it is appropriate to choose a new coordinate system rotating the original one used in (1) by the angle $\theta$ in the $x y$ plane [20]. The rotation angle $\theta$ is determined by the requirement $\left\langle S^{x^{\prime}}\right\rangle=0$ in the new coordinate system.

The retarded Green's function to be calculated is defined as

$$
G_{i j}(t)=\ll S_{i}^{+}(t) ; S_{j}^{-}(0) \gg
$$

where $S^{+}$and $S^{-}$are the spin- $\frac{1}{2}$ operators in the rotated system. On introducing the twodimensional Fourier transform $G_{n_{i} n_{j}}\left(\mathbf{k}_{\|}, \omega\right)$, one has the following form:

$$
\ll S_{i}^{+} ; S_{j}^{-} \gg_{\omega}=\frac{\sigma}{N^{\prime}} \sum_{\mathbf{k}_{\|}} \exp \left(i \mathbf{k}_{\|}\left(\mathbf{r}_{i}-\mathbf{r}_{j}\right)\right) G_{n_{i} n_{j}}\left(\mathbf{k}_{\|}, \omega\right)
$$

where $N^{\prime}$ is the number of sites in any of the lattice planes, $\mathbf{r}_{i}$ and $n_{i}$ represent the position vectors of site $\mathrm{i}$ and the layer index, respectively, $\mathbf{k}_{\|}=\left(k_{x}, k_{y}\right)$ is a two-dimensional wave vector parallel to the surface. The summation is taken over the Brillouin zone.

As a result the equation of motion for the Green's function in Eq. (3) of the ferroelectric thin film for $T \leq T_{c}$ has the following matrix form:

$$
\mathbf{H}(\omega) \mathbf{G}\left(\mathbf{k}_{\|}, \omega\right)=\mathbf{R},
$$


where $\mathbf{H}$ can be expressed as:

$$
\mathbf{H}=\left(\begin{array}{ccccccc}
\omega-V_{1} & k_{1} & 0 & 0 & 0 & 0 & \ldots \\
k_{2} & \omega-V_{2} & k_{2} & 0 & 0 & 0 & \ldots \\
0 & k_{3} & \omega-V_{3} & k_{3} & 0 & 0 & \ldots \\
\vdots & \vdots & \vdots & \vdots & \vdots & \vdots & \ddots \\
0 & 0 & 0 & 0 & 0 & k_{N} & \omega-V_{N}
\end{array}\right)
$$

with

$$
\begin{aligned}
k_{n} & =J_{b} \sigma_{n} \sin ^{2} \theta_{n}, \quad n=1, \ldots, N, \\
V_{1} & =2 \Omega_{s} \sin \theta_{1}+\frac{1}{2} \sigma_{1} J_{s} \cos ^{2} \theta_{1}-\frac{\sigma_{1} J_{s}}{4} \sin ^{2} \theta_{1} \gamma\left(\mathbf{k}_{\|}\right)+J_{b} \sigma_{2} \cos ^{2} \theta_{2}, \\
V_{2} & =2 \Omega_{b} \sin \theta_{2}+\frac{1}{2} \sigma_{2} J_{b} \cos ^{2} \theta_{2}-\frac{\sigma_{2} J_{b}}{4} \sin ^{2} \theta_{b} \gamma\left(\mathbf{k}_{\|}\right)+J_{s} \sigma_{1} \cos ^{2} \theta_{+} J_{b} \sigma_{3} \cos ^{2} \theta_{3}, \\
V_{n} & =2 \Omega_{n} \sin \theta_{n}+\frac{1}{2} \sigma_{n} J_{b} \cos ^{2} \theta_{n}-\frac{\sigma_{n} J_{n}}{4} \sin ^{2} \theta_{n} \gamma\left(\mathbf{k}_{\|}\right)+J_{n-1} \sigma_{n-1} \cos ^{2} \theta_{n-1} \\
& +J_{n+1} \sigma_{n+1} \cos ^{2} \theta_{n+1}, \\
V_{N} & =2 \Omega_{s} \sin \theta_{N}+\frac{1}{2} \sigma_{N} J_{s} \cos ^{2} \theta_{N}-\frac{\sigma_{N} J_{s}}{4} \sin ^{2} \theta_{N} \gamma\left(\mathbf{k}_{\|}\right)+J_{b} \sigma_{N-1} \cos ^{2} \theta_{N-1}, \\
\gamma\left(\mathbf{k}_{\|}\right) & =\frac{1}{2}\left(\cos \left(k_{x} a\right)+\cos \left(k_{y} a\right)\right) .
\end{aligned}
$$

Here we have introduced the notations $J_{1} \equiv J_{N}=J_{s}, J_{n}=J_{b}$ for $n=2,3,4, \ldots, N-1$, $\Omega_{1}=\Omega_{N}=\Omega_{s}, \Omega_{n}=\Omega_{b}(\mathrm{n}=2,3,4, \ldots, \mathrm{N}-1), J_{0}=J_{N+1}=0$. The quantity $\sigma(T)$ is the relative polarization in the direction of the mean field and is equal to $2\left\langle S^{z^{\prime}}\right\rangle$. For the rotation angle $\theta$ we have the following two solution in the generalized Hartree-Fock approximation:

$$
\begin{array}{ll}
\text { 1. } \cos \theta=0, \quad \text { i.e. } \quad \theta=\frac{\pi}{2}, & \text { if } T \geq T_{c} ; \\
\text { 2. } \sin \theta=\frac{4 \Omega}{\sigma J}=\frac{\sigma_{c}}{\sigma}, & \text { if } \quad T \leq T_{c} .
\end{array}
$$

In order to obtain the solutions of the matrix equation (44), we introduce the twodimensional column matrices, $\mathbf{G}_{m}$ and $\mathbf{R}_{m}$, where the elements are given by $\left(\mathbf{G}_{n}\right)_{m}=G_{m n}$ and $\left(\mathbf{R}_{n}\right)_{m}=\sigma_{n} \delta_{m n}$, so that Eq. (41) yields

$$
\mathbf{H}(\omega) \mathbf{G}_{n}=\mathbf{R}_{n}
$$

From Eq. (5),$G_{n n}(\omega)$ is obtained as:

$$
G_{n n}(\omega)=\frac{\left|H_{n n}(\omega)\right|}{|H(\omega)|} .
$$


The quantity $\left|H_{n n}(\omega)\right|$ is the determinant made by replacing the $n$-th column of the determinant $|H(\omega)|$ by $R_{n}$. The poles $\omega_{n}$ of the Green's function $G_{n n}(\omega)$ can be calculated by solving $|H(\omega)|=0$.

The relative polarization of the of the $n$-th layer is given by

$$
\sigma_{n}=\left(\frac{\sigma_{n} J_{n}}{2 N} \sum_{\mathbf{k}_{\|}} \frac{1-0.5 \sin ^{2} \theta_{n} \gamma\left(\mathbf{k}_{\|}\right)}{\omega_{n}} \operatorname{coth} \frac{\omega_{n}}{2 T}\right)^{-1} .
$$

The last equation has to be calculated numerically. Due to the assumption of symmetrical surfaces, there are $\frac{1}{2} \mathrm{~N}$ layer polarizations, which have to be solved self-consistently. In order to obtain the dependence of the Curie temperature $T_{C}$ on the film thickness $N$, we let all $\sigma$ 's be zero in Eq. (77) and solve the expressions self-consistently.

\section{NUMERICAL RESULTS AND DISCUSSION}

In this section we present the numerical results based on our theoretical calculations taking the typical the bulk parameter $J_{b}=495 \mathrm{~K}$ and $\Omega_{b}=20 \mathrm{~K}$. We have calculated the temperature dependence of the polarization defined by

$$
\sigma \equiv<S^{z}>=\frac{1}{N} \sum_{n} \sigma_{n}(T) \cos \theta_{n}
$$

and the spin-wave frequencies of thin films for different values of the exchange interaction constants. One has to solve self-consistently the $\frac{1}{2} \mathrm{~N}$ coupled Eqs. (17) to obtain the layer polarization from the surface to half of the film (the other half is symmetric due to the assumption of identical surfaces). The numerical results expose some interesting and novel characteristics in the polarization and the spin-wave energies in comparison to the case of $\mathrm{FE}$ thin films without defects. To characterize the complete ferroelectric system both quantities, the polarization $\sigma$ and the spin-wave energy $\omega$, respectively are averaged over the $N$ layers. The results for film thickness $N=9$ and different exchange interaction parameters in the defect layers $J_{d}$ are presented in Fig. 11 and Fig. 2. Let us firstly consider the case that the middle layer is defect layer. For example, it can be originated by localized vacancies or impurities with smaller radii and larger distances between them in comparison to the host material. It is reasonable to assume that the exchange interaction $J_{d}$ is smaller than the value of the bulk interaction $J_{b}=495 \mathrm{~K}$ and has the value $J_{5}=J_{d}=300 \mathrm{~K}$ (compare Figs. 1, 2 curve 1). The polarization (respectively the spin-wave energy) is smaller than 
the case without defects, $J_{5}=J_{b}$ (see Figs. 1, 2 curve 2). The polarization decreases with increasing temperature to vanish at the critical temperature $T_{C}$ of the thin film. The critical temperature decreases due to the smaller $J_{d}$ value. Pontes et al. [3] carried out dielectric and Raman spectroscopy studies and obtained that with addition of $\mathrm{Sr}$ to $\mathrm{PbTiO}_{3}$, the phase-transition temperature decreases with the increasing Sr concentration.

For the case where $J_{5}=J_{d}=1000 K$ (Figs. 1 2, curve 3), i.e. $J_{d}$ is larger than the value of the bulk interaction constant $J_{b}$, (for example when the impurities have a larger radius compared with the constituent ions) the polarization (respectively the spin-wave energy) is larger than the case without defects, $J_{5}=J_{b}$. $T_{C}$ of the film is enhanced in comparison to the bulk value without defects due to the presence of larger $J_{d}$ values. This is the opposite behavior compared to the case of $J_{d}=300 K, J_{d}<J_{b}$. Of course, in the case of both strong and small $J_{d}$ values, thin film polarizations tend to the $3 \mathrm{D}$ one when $n$ increases, since the excitation spectrum varies from $2 \mathrm{D}$ to $3 \mathrm{D}$ behavior with thickness. The second case, where $J_{d}>J_{b}$ could explain the experimentally obtained increase in polarization by the substitution of impurities, such as $\mathrm{Nd}, \mathrm{V}, \mathrm{W}$ and $\mathrm{Nb}$ in the layers of BTO films [6, 7, 8] or by increasing Ca contents in SBT thin films [11].

From Figs. 1] 2 one can observe that the polarization, the spin-wave energies and the critical temperature of the FE phase transition are increased or decreased due to different exchange interactions in the defect layers. Our results are in qualitatively agreement with the experimental data of Noguchi et al. [15]. They have studied SBT films $\left(T_{C}=295^{\circ} C\right)$ by the substitution of rare earth cations of $\mathrm{La}, \mathrm{Ce}, \mathrm{Pr}, \mathrm{Nd}$ and $\mathrm{Sm}$ as well as Bi at the A site ( $\mathrm{Sr}$ site) with Sr vacancies and have shown, that La modification induces soft behavior (lower $E_{c}$ and lower $\sigma_{r}$ ), while a large amount of $\mathrm{Nd}$ and $\mathrm{Sm}$ substitution results in a very high $E_{c}$ (hard), as a result of defect engineering of both $\mathrm{Sr}$ and oxide vacancies. For not only SBT, but also other bismuth layer-structured FE (BLSF's) $T_{C}$ is strongly influenced by $r_{i}$ of A-site cations, and BLSF's with smaller A-site cations $\left(\mathrm{Ca}^{2+}\right)$ tend to show a higher $T_{C}\left(420^{0} \mathrm{C}\right)$. The same amount of larger $\mathrm{Ba}^{2+}$ brings about a relaxation of $\mathrm{FE}$ distortions and resulted in a decrease in $T_{C}$ to $120^{\circ} \mathrm{C}$. The substitution of La led to a marked decrease in $T_{C}$ to $180^{\circ} \mathrm{C}$ $(x=0.5)$, because the induced A-site vacancies weaken the coupling between neighboring $\mathrm{BO}_{6}$ octahedral [5, 15]). This result corresponds in our calculations to the case of smaller values of the interaction constant in the defect layer $J_{d}<J_{b}$. For La-modified $\mathrm{PbTiO}_{3}, T_{C}$ decreased significantly, too, with an increase in La content [28]. For Bi-SBT $T_{C}$ rose strongly 
to $405^{\circ} \mathrm{C}(x=0.2)$ [15]. The increase in $T_{C}$ by Bi substitution is an opposite tendency to that in the case for La-SBT. The dominant role plays here the bonding characteristics with oxide ions. The influence of the orbital hybridization on $T_{C}$ is very large, and Bi substitution resulted in a higher $T_{C}$ [15]. This experimental result can be described qualitatively good using in our model exchange interaction parameters for the defect layer $J_{d}>J_{b}$.

In Figs. 3, 4 is shown the influence of the surface exchange interaction constant $J_{s}$ on the polarization and spin-wave energy by $J_{4}=J_{d}=$ const for a thin film with $N=7$ layers. It can be seen that the two quantities are enhanced with increasing of $J_{s}$. The critical temperature $T_{C}$ increases, too.

The polarization is depending, too, on that how many inner layers are defect. This is shown in Figs. 5, 6 for a thin film with $N=7$ layers. In Fig. 51 is demonstrated that for $J_{d}=200 K$ with increasing of the number of defect layers (curve $1-J_{4}=J_{d}=J_{b}$, curve 2 - $J_{4}=J_{d}=200 \mathrm{~K}$, curve $\left.3-J_{3}=J_{4}=J_{5}=J_{d}=200 \mathrm{~K}\right)$ the polarization and the critical temperature decreases. The opposite behavior can be seen in Fig. 6, where $J_{d}=1000 \mathrm{~K}$. With increasing of the number of defect layers the polarization and $T_{C}$ increase. The spinwave energies are reduced in the first case and enhanced in the second case.

We have also studied a ferroelectric thin film whit $N=9$ layers where one layer, the middle one is defect and the spins are ordered antiparallel, the defect layer is antiferroelectric, $J_{5}=J_{d}=-1000 K$ (Fig. 7) curve 2) and $J_{5}=J_{d}=-300 K$ (Fig. 8, curve 2). The polarization and $T_{C}$ of the film are smaller in comparison with the case where the middle layer is defect, but with ferroelectric order. The spin-wave energy of the thin film is also reduced when $J_{5}=J_{d}=-1000 K$. Such a sublattice model with ferroelectric and antiferroelectric layers where one or more layers are defect will be studied in a next paper.

\section{CONCLUSIONS}

In conclusions, based on a modified transverse Ising model and using a Green's function technique, the polarization, the spin-wave energies and the phase transition temperature for ferroelectric thin films with structural defects are calculated. The dependence on temperature, film thickness and interaction constants is discussed. It is shown that defect layers in FE thin films, layers with impurities or vacancies, with dislocations, can induce strong increasing or decreasing of the polarization, the spin-wave energies and of the critical tem- 
perature of FE phase transition due to different exchange interactions in the defect layers. The results are in good agreement with the experimental data for different ferroelectrics.

\section{Acknowledgments}

One of us (J. M. W.) is grateful to the Deutsche Forschungsgemeinschaft for financial support. This work is supported by the SFB 418. Further, we thank Dr. D. Hesse and Dr. M. Alexe, Max Planck Institute of Microstructure Physics, Halle for fruitful and exciting discussions on experimental details. 
[1] S.H. Kim, J.S. Yang, C.Y. Koo, J.H. Yeom, E. Yoon, C.S. Hwang, J.S. Park, S.G. Kang, D.J. Kim and J. Ha, Jpn. J. Appl. Phys. 42, 5952 (2003).

[2] T. Tohma, H. Masumoto and T. Goto, Jpn. J. Appl. Phys. 42,6969 (2003).

[3] F.M. Pontes, S.H. Leal, E.R. Leite, E. Longo, P.S. Pizani, A.J. Chiquito and J.A. Varela, J. Appl. Phys. 96, 1192 (2004).

[4] M.W. Chu, I. Szafraniak, R. Scholz, C. Harnagea, D. Hesse, M. Alexe and U. Goesele, Nature Materials 3, 87 (2004).

[5] T. Sakai, T. Watanabe, H. Funakubo, K. Sarro and M. Osada, Jpn. J. Appl. Phys. 42, 166 (2003).

[6] J.S. Kim, S.S. Kim and J.K. Kim, Jpn. J. Appl. Phys. 42, 6486 (2003).

[7] E.K. Choi, S.S. Kim, J. K. Kim, J.C. Bae, W.J. Kim, Y.I. Lee and T.K. Song, Jpn. J. Appl. Phys. 43, 237 (2004).

[8] T. Watanabe, T. Kojima, H. Uchida, I. Okada and H. Funakubo, Jpn. J. Appl. Phys. 43, L309 (2003).

[9] Y. Noguchi, M. Miyayama, K. Oikawa, T. Kamiyama, M. Osada and M. Kakihana, Jpn. J. Appl. Phys. 41, 7062 (2002).

[10] K. Aizawa and H. Ishiwara, Jpn. J. Appl. Phys. 42, L840 (2003).

[11] R.R. Das, P. Bhattacharya, W. Perez and R.S. Katiyar, Jpn. J. Appl. Phys. 42, 162 (2003).

[12] D. Berlincourt, D. Curran and H. Jaffe: Physical Acoustics, ed. W. Cady (Academic, New York, 1964) Vol. 1.

[13] J.F. Scott: Ferroelectric Memories (Springer, Berlin, 2000).

[14] Y. Shimakawa and Y. Kubo, Appl. Phys. Lett. 74, 1904 (1999).

[15] Y. Noguchi, M. Miyayama and T. Kudo, Phys. Rev. B 63, 214102 (2001).

[16] L.H. Robins, D.L. Kaiser, L.D. Rotter, P.K. Schenck, G.T. Stauf, and D. Rytz, J. Appl. Phys. 76, 7487 (1984).

[17] D.A. Tenne, et al Phys. Rev. B 69, 174101 (2004).

[18] D. Dourelly, D. Hone and V. Jaccarino, Phys. Rev. Lett. 65, 2286 (1990).

[19] R.N. Costa Filho, U.M.S. Costa and M.G. Cottam, J. Magn. Magn. Mater. 213, 195 (2000).

[20] J.M. Wesselinowa, phys. stat. sol. (b) 223, 737 (2001). 
[21] M. Pantic, M. Manojlovic, M. Skrinjar, M. Pavkov, D. Kapor and S. Stojanovic, Int. J. Mod. Phys. B 18, 1537 (2004).

[22] X.L. Zhang, B.Y. Gu and K.Q. Chen, Physics Lett. A 316, 107 (2003).

[23] V.S. Vikhin and T.I. Maksimova, Ferroel. 299, 43 (2004).

[24] O. Dieguez, S. Tinte, C. Bungaro, J.B. Neaton, K.M. Rabe and D. Vanderbilt, Phys. Rev. B 69, $212101(2004)$

[25] N.A. Pertsev, A.G. Zembilgotov and A.K. Tagantsev, Phys. Rev. Lett. 80, 1988 (1998).

[26] S.P. Alpay, I.B. Misirlioglu, A. Sharma and Z.G. Ban, J. Appl. Phys. 95, 8118 (2004).

[27] S.P. Alpay, I.B. Misirlioglu, V. Nagarajan and R. Ramesh, Appl. Phys. Lett. 85, 2044 (2004).

[28] T.Y Kim and H.M. Jang, Appl. Phys. Lett. 77, 3824 (2000). 


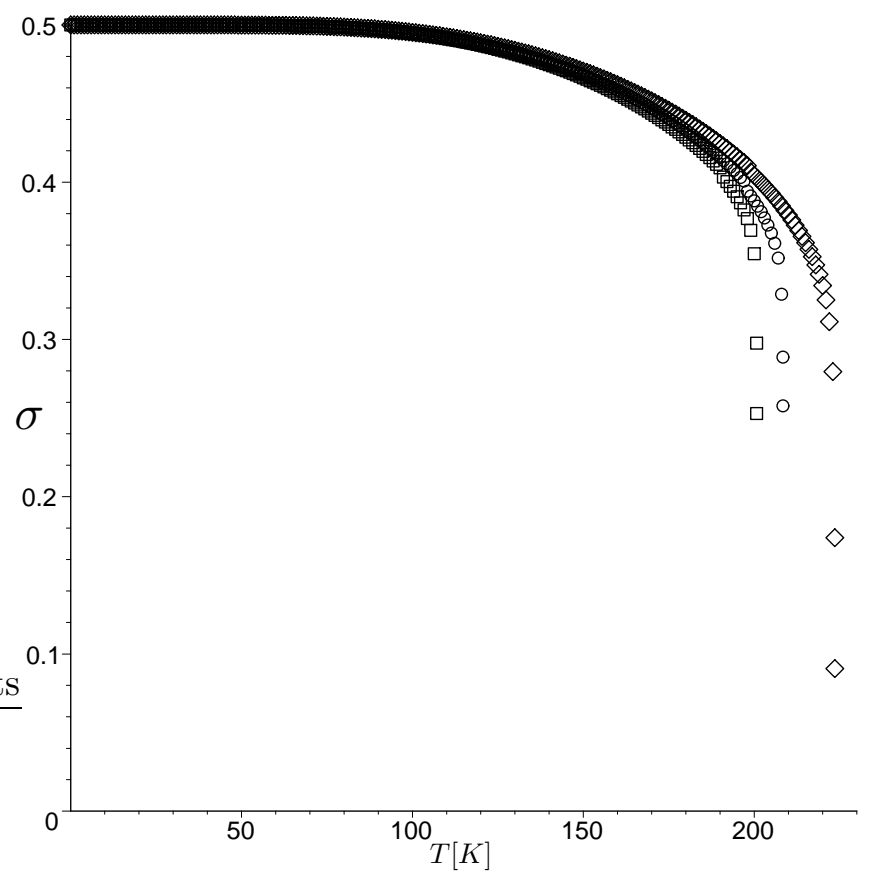

FIG. 1: Temperature dependence of the polarization $\sigma$ for a FE thin film with $J_{b}=495 K, \Omega_{b}=$ $20 K, J_{s}=900 K, \Omega_{s}=\Omega_{b}, N=9$ and different $J_{d}$-values: (1) $J_{d}=300 \mathrm{~K}, T_{c}=201 K(\square),(2) 495 K$, $T_{c}=208 K(\circ),(3) 1000 K, T_{c}=224 K(\diamond)$. 


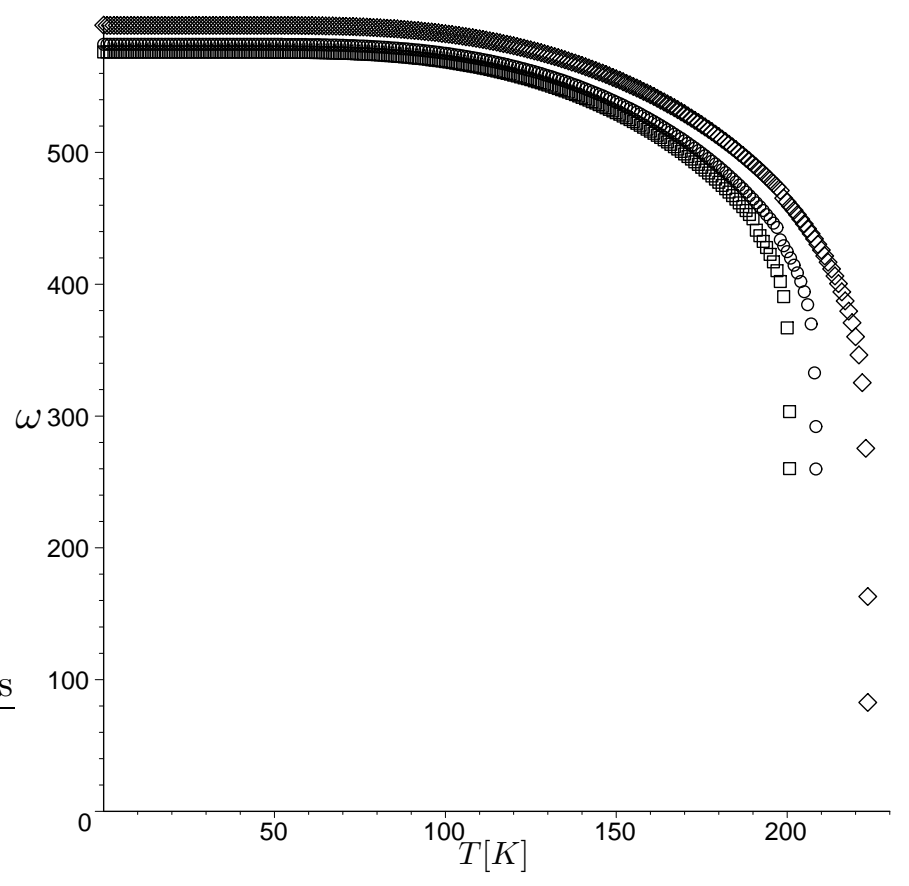

FIG. 2: Temperature dependence of the spin-wave energy $\omega\left[\mathrm{cm}^{-1}\right]$ for a FE thin film with $J_{b}=$ $495 K, \Omega_{b}=20 K, J_{s}=900 K, \Omega_{s}=\Omega_{b}, N=9$ and different $J_{d}$-values: (1) $J_{d}=300 K(\square),(2)$ $495 K(\circ),(3) 1000 K(\diamond)$.

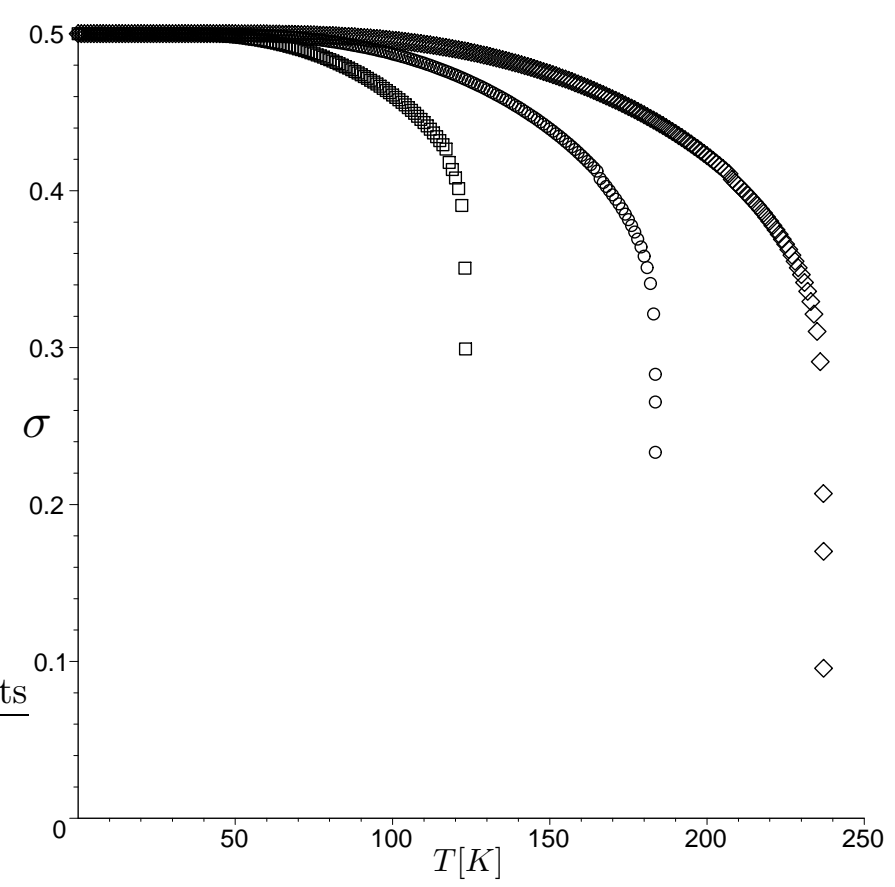

FIG. 3: Temperature dependence of the polarization $\sigma$ for $J_{b}=495 K, \Omega_{b}=20 K, J_{d}=1000 K$, $\Omega_{s}=\Omega_{b}, N=7$ and different $J_{s}$-values: (1) $J_{s}=200 K, T_{c}=123 K(\square),(2) 500, T_{c}=184 K$ (o), (3) $1000 K, T_{c}=237 K(\diamond)$. 


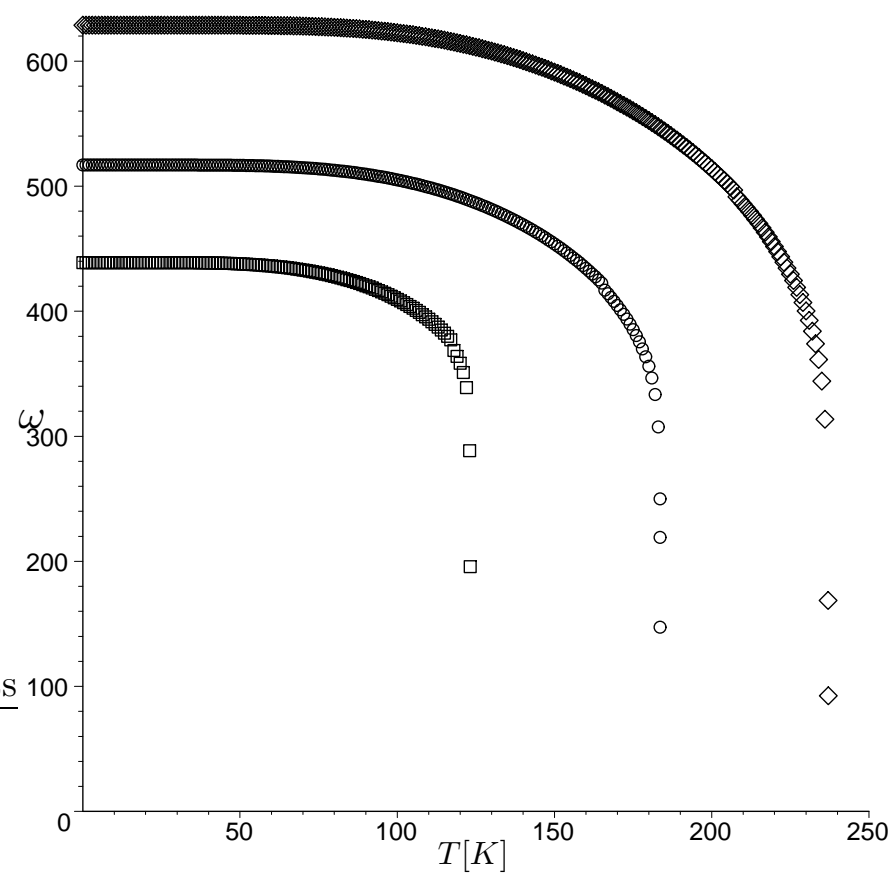

FIG. 4: Temperature dependence of the spin-wave energy $\omega\left[\mathrm{cm}^{-1}\right]$ for $J_{b}=495 K, \Omega_{b}=20 K$, $J_{d}=1000 K, \Omega_{s}=\Omega_{b}, N=7$ and different $J_{s}$-values: (1) $J_{s}=200 K(\square),(2) 500 K(\circ),(3) 1000 K$ $(\diamond)$.

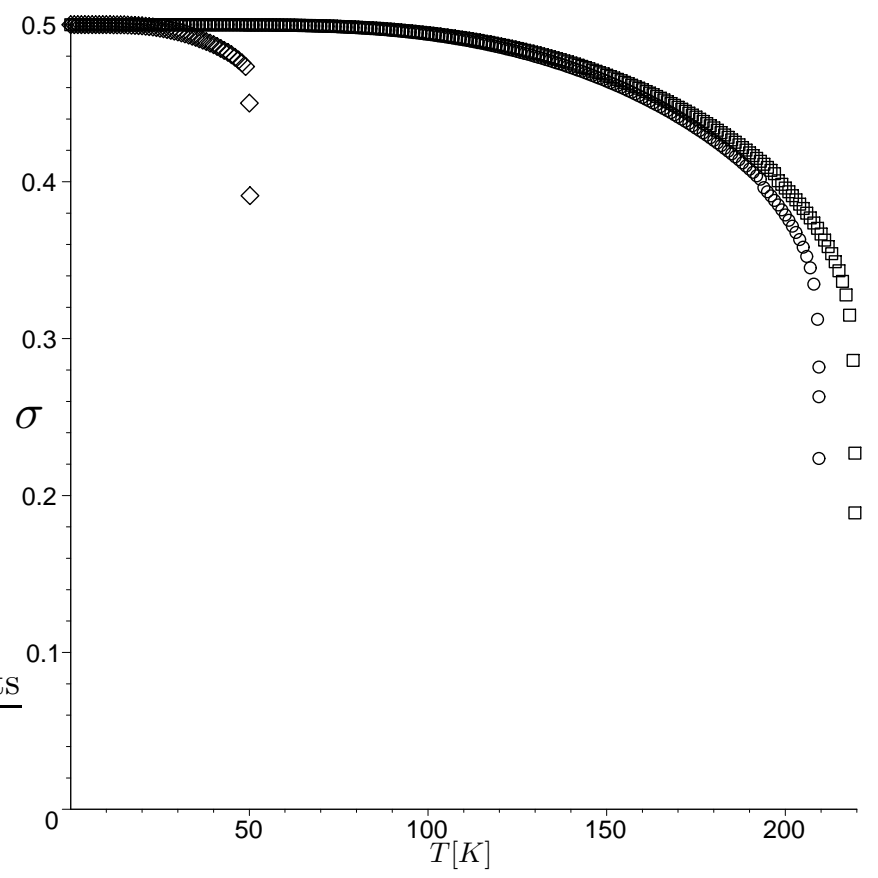

FIG. 5: Temperature dependence of the polarization $\sigma$ for $J_{b}=495 K, \Omega_{b}=20 K, J_{s}=900 K$, $\Omega_{s}=\Omega_{b}, N=7$ and different defect layers: (1) $J_{4}=J_{b}=495 K, T_{c}=220 K(\square)$, (2) $J_{4}=J_{d}=$ $200 K, T_{c}=209 K(\circ),(3) J_{3}=J_{4}=J_{5}=J_{d}=200 K, T_{c}=50 K(\diamond)$. 


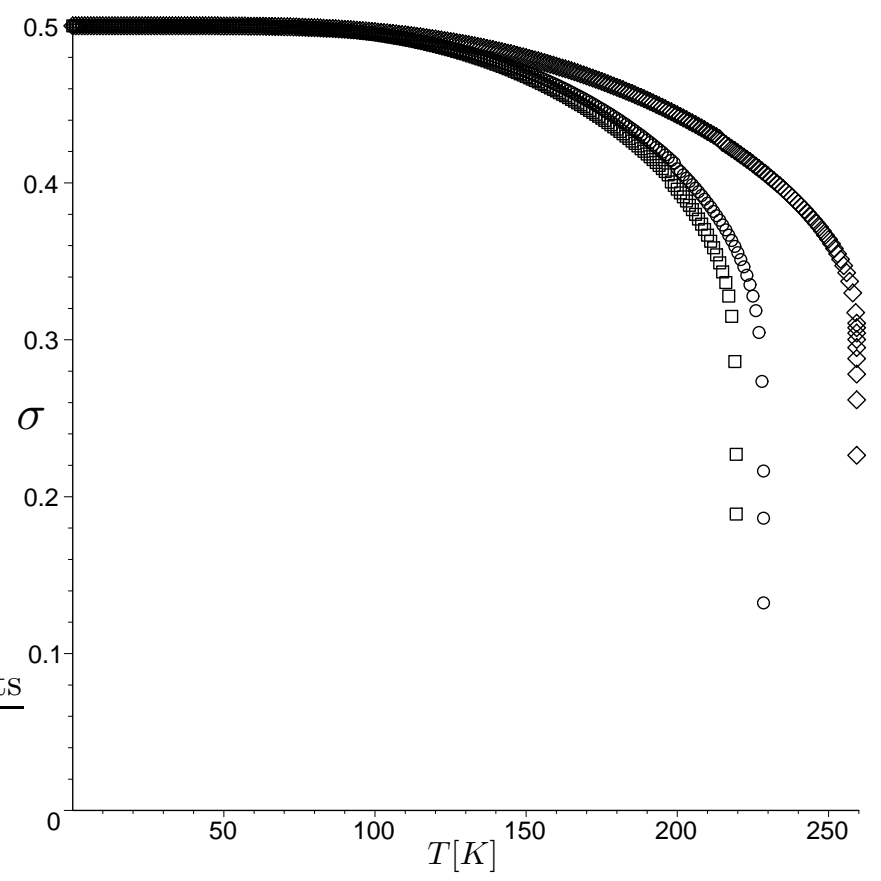

FIG. 6: Temperature dependence of the polarization $\sigma$ for $J_{b}=495 K, \Omega_{b}=20 K, J_{s}=900 K$, $\Omega_{s}=\Omega_{b}, N=7$ and different defect layers: (1) $J_{4}=J_{b}=495 K, T_{c}=220(\square)$, (2) $J_{4}=J_{d}=$ $1000 K, T_{c}=229(\circ),(3) J_{3}=J_{4}=J_{5}=1000 K, T_{c}=259 K(\diamond)$.

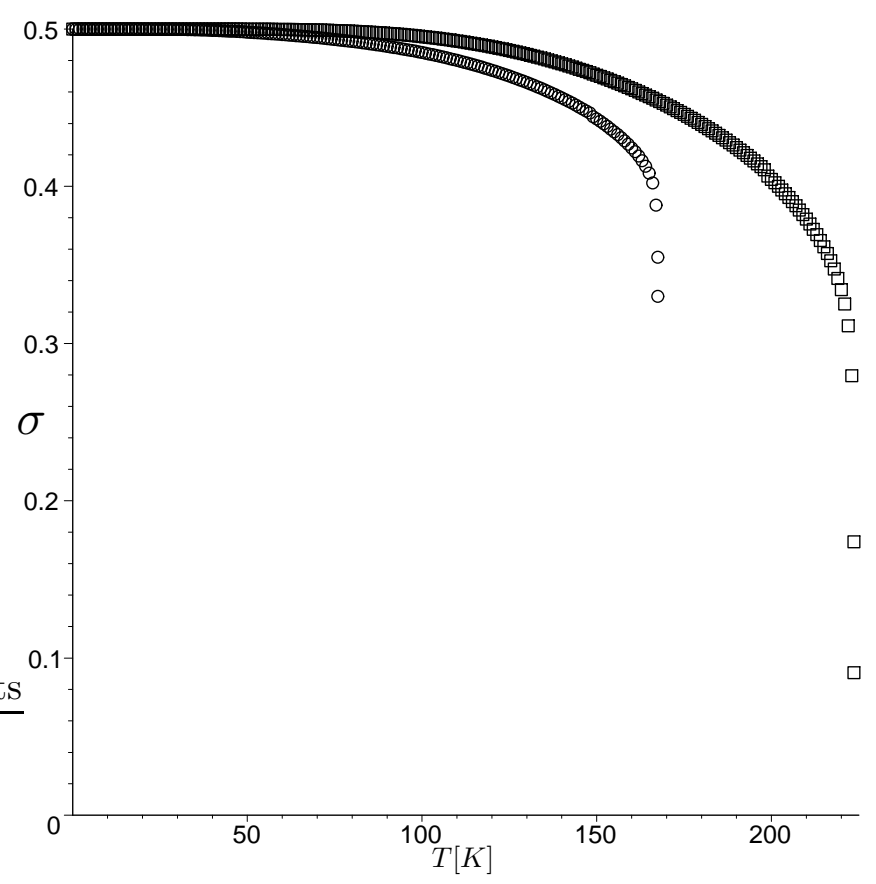

FIG. 7: Temperature dependence of the polarization $\sigma$ with $J_{b}=495 K, \Omega_{b}=20 K, J_{s}=900 K$, $\Omega_{s}=\Omega_{b}, N=9$ and an antiferroelectric defect layer: (1) $J_{d}=1000 K, T_{c}=223 K(\square)$, (2) $-1000 K, T_{c}=168 K(\circ)$. 


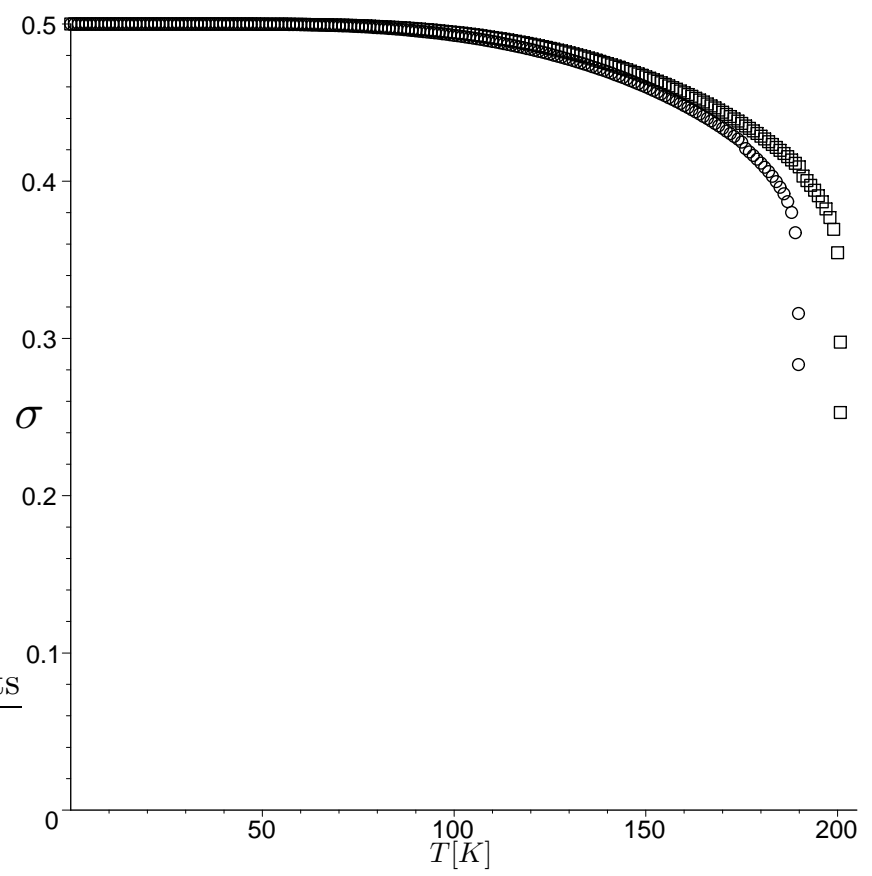

FIG. 8: Temperature dependence of the polarization $\sigma$ with $J_{b}=495 K, \Omega_{b}=20 K, J_{s}=900 K$, $\Omega_{s}=\Omega_{b}, N=9$ and an antiferroelectric defect layer: (1) $J_{d}=300 K, T_{c}=201 K(\square),(2)$ $-300 K, T_{c}=190 K(\circ)$. 